\section{Wo läuft welche Studie?}

Bei $40-70 \%$ aller Studien zur ParkinsonErkrankung weltweit tritt wegen einer zu schleppenden Rekrutierung von Patienten eine Verzögerung auf. Die Michael J. Fox Foundation hat sich zum Ziel gesetzt, das zu optimieren. Der Fox Trial Finder (https://foxtrialfinder.michaeljfox.org/) soll Studienteams, Zuweiser und Patienten zueinander bringen. Wie Professor Karla Eggert, Marburg, berichtete, will sich auch das Kompetenznetz Parkinson mit seinem Studiennetzwerk daran beteiligen. Patienten und gesunde Probanden melden sich zunächst anonym mit ihren Basisdaten an, Studienteams können ihre registrierten Studien eingeben. „Es ist wichtig, dass auch wir aktiv diese Datenbank speisen", betonte Eggert. Zunächst sollte dazu das Studienzentrum in Marburg kontaktiert werden (www.kompetenznetz-parkinson.de).

Friederike Klein

Eggert K: Symposium „Medikamentöse Innovationen der Parkinson-Therapie". Deutscher Parkinson-Kongress 2013, Würzburg, 14.3. 2013

\section{Patienten gesucht!}

Viele Kohortenstudien weltweit untersuchen den Verlauf der Parkinson-Erkrankung (PD) auf der Suche nach Prädiktoren und prognostisch relevanten Markern. Wie Professor Richard Dodel, Marburg, berichtete, wird aktuell auch in Deutschland in 19 Zentren für die Studie "Langzeitbeobachtung dementieller Symptome und cognitiver Parameter sowie Anwendbarkeit neuer prognostischer Marker bei der Parkinson-Erkrankung" (LANDSCAPE) rekrutiert. An der longitudinalen Kohortenstudie sollen 250 Parkinson-Patienten ohne und ebenso viele mit Demenz sowie 200 Patienten mit leichter kognitiver Einschränkung $(\mathrm{MCl})$ teilnehmen. Ziel ist, genetische und biochemische Biomarker für Demenzentwicklung und -verlauf aufzudecken, den Verlauf in der Bildgebung zu verfolgen und mit neuropsychologischen Batterien die Veränderung der kognitiven Plastizität zu beobachten. Für die Studie werden dringend noch Patienten mit Lewy-Körperchen-Demenz gesucht. Weitere Informationen zur Studie finden sich im Internet unter Kompetenznetzwerk Demenzen (www.knd-demenzen.de, Menü Verbünde/Verbund Parkinson).

Friederike Klein

Dodel R: Vortrag im Rahmen des Symposiums "Klinische Studien". Deutscher Parkinson-Kongress 2013, Würzburg, 15.3.2013

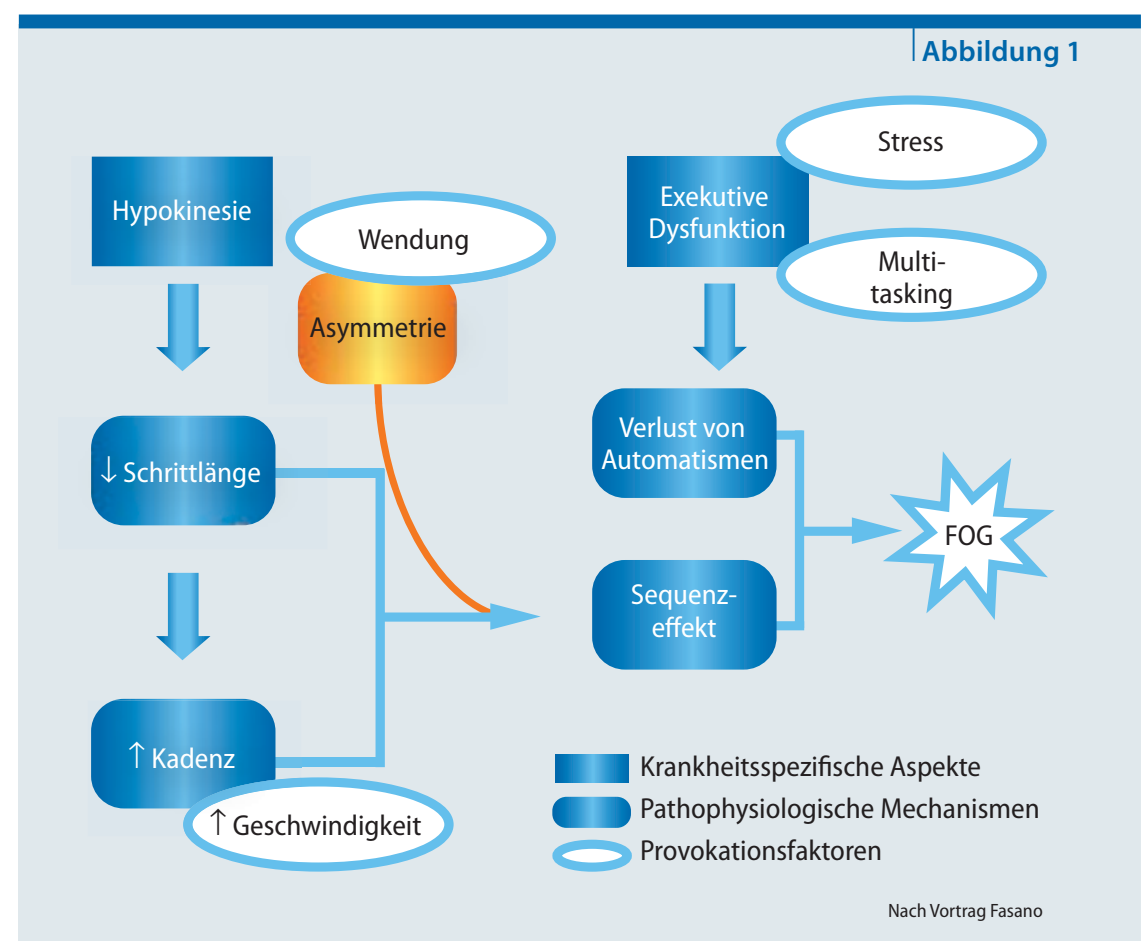

Freeze-of-Gait (FOG) - ein komplexes Geschehen.

dem Boden mobil, ist aber teuer, verlangsamt initial die Gehstrecke eher und ist für die meisten Patienten nicht geeignet, urteilte Caballos-Baumann. Akustische Cues, Musik oder Metronom sind beispielsweise als MP3-Player unauffällig und können Spaß machen. Allerdings fallen damit Umgebungsgeräusche weg und es hilft auch nicht jeder Takt gleichermaßen.

Letztlich empfiehlt der Experte: Cues ausprobieren und das, was Erfolg verspricht, trainieren. Als gelungenes Beispiel nannte Caballos-Baumann das zweiwöchige Münchener Anti Freezing Training (MAFT). Dreimal 30 Minuten pro Woche mit frei wählbaren Cues verbesserten das On-Freezing signifikant gegenüber einer Wartelistengruppe [6].

\section{Teures Spezialgerät oder Eigenbau?}

Mit teuren Spezialgeräten hat CaballosBaumann keine besonders überzeugenden Erfahrungen gemacht - ob der bereits erwähnte Anti-Freezing-Stock, eine Anti-Freezing-Brille mit mitlaufenden Streifen am Rand des Blickfelds oder Spezialrollator mit Laserlinie und Taktgeber, der in Großbritannien für
$16.000 €$ angeboten wird. „Das kann man doch auch selbst zusammenbauen", meinte Caballos-Baumann zum letzten Vorschlag. Eine seiner Patientinnen kommt beispielsweise sehr gut mit einem Laserpointer mit strichförmigem Licht aus dem Baumarkt zurecht. Und an den Rollator kann man einen Luftballon binden, gegen den die Patienten treten sollen. „Das funktioniert ganz gut", sagte Caballos-Baumann, „und im Gegensatz zum Laser auch bei Sonnenlicht."

Friederike Klein

1. Fietzek WM et al. Basal Ganglia 2013; 3: 64 (Abstract P65)]

2. Fasano A et al. Mov Disord 2011; 26: 844-51

3. Chee R et al. Brain. 2009; 132: $2151-60$

4. Amboni M et al. Mov Disord 2012; 27:1 53643

5. Espay AJ et al. Neurology 2012; 78: 454-7

6. Ziegler K et al. Mov Disord 2010; 25: 1012-8

Vorträge „Therapie des Freezing" (Caballos-Baumann A) und "Pathophysiologie des Freezing" (Fasano A) im Rahmen des Symposiums „Pathophysiologie der Gangstörungen", Deutscher Parkinson-Kongress 2013, Würzburg, 13.-15.3. 2013 